\title{
Oblivious Transfers and Privacy Amplification
}

\author{
Gilles Brassard * and Claude Crépeau ${ }^{\star \star}$ \\ Département IRO, Université de Montréal \\ C.P. 6128 , succursale centre-ville \\ Montréal (Québec), Canada H3C 3J7 \\ email: $\{$ brassard,crepeau\}@iro.umontreal.ca
}

\begin{abstract}
Assume $\mathcal{A}$ owns two secret $k$-bit strings. She is willing to disclose one of them to $\mathcal{B}$, at his choosing, provided he does not learn anything about the other string. Conversely, $\mathcal{B}$ does not want $\mathcal{A}$ to learn which secret he chose to learn. A protocol for the above task is said to implement One-out-of-two String Oblivious Transfer, denoted $\left(\begin{array}{l}2 \\ 1\end{array}\right)-\mathrm{OT}^{k}$. This primitive is particularly useful in a variety of cryptographic settings. An apparently simpler task corresponds to the case $k=1$ of two one-bit secrets: this is known as One-out-of-two Bit Oblivious Transfer, denoted $\left(\begin{array}{l}2 \\ 1\end{array}\right)$-OT. We address the question of reducing $\left(\begin{array}{l}2 \\ 1\end{array}\right)-\mathrm{OT}^{k}$ to $\left(\begin{array}{l}2 \\ 1\end{array}\right)$-OT. This question is not new: it was introduced in 1986. However, most solutions until now have implicitly or explicitly depended on the notion of self-intersecting codes. It can be proved that this restriction makes it asymptotically impossible to implement $\left(\begin{array}{l}2 \\ 1\end{array}\right)-O T^{k}$ with fewer than about $3.5277 k$ instances of $\left(\begin{array}{l}2 \\ 1\end{array}\right)$-OT. The current paper introduces the idea of using privacy amplification as underlying technique to reduce $\left(\begin{array}{l}2 \\ 1\end{array}\right)-O T^{k}$ to $\left(\begin{array}{l}2 \\ 1\end{array}\right)$-OT. This allows for more efficient solutions at the cost of an exponentially small probability of failure: it is sufficient to use slightly more than $2 k$ instances of $\left(\begin{array}{l}2 \\ 1\end{array}\right)-\mathrm{OT}$ in order to implement $\left(\begin{array}{l}2 \\ 1\end{array}\right)-\mathrm{OT}^{k}$. Moreover, we show that privacy amplification allows for the efficient implementation of $\left(\begin{array}{l}2 \\ 1\end{array}\right)-\mathrm{OT}^{k}$ from generalized versions of $\left(\begin{array}{l}2 \\ 1\end{array}\right)$-OT that would not have been suitable for the earlier techniques based on self-intersecting codes. An application of this more general reduction is given.
\end{abstract}

Key Words: Information-Theoretic Security, Reduction Between Protocols, Oblivious Transfer, Privacy Amplification.

\footnotetext{
* Supported in part by Canada's NSERC, The Canada Council and Québec's FCAR.

** Supported in part by Québec's FCAR and Canada's NSERC.
} 


\section{Introduction}

One-out-of-two String Oblivious Transfer, denoted $\left(\begin{array}{l}2 \\ 1\end{array}\right)-\mathrm{OT}^{k}$, is a primitive that originates with [Wie70] (under the name of "multiplexing"), a paper that marked the birth of quantum cryptography. According to this primitive, one party $\mathcal{A}$ owns two secret $k$-bit strings $w_{0}$ and $w_{1}$, and another party $\mathcal{B}$ wants to learn $w_{c}$ for a secret bit $c$ of his choice. $\mathcal{A}$ is willing to collaborate provided that $\mathcal{B}$ does not learn any information about $w_{\bar{c}}$, but $\mathcal{B}$ will only participate if $\mathcal{A}$ cannot obtain information about $c$. Independently from [Wie70] but inspired by [Rab81], a natural restriction of this primitive was introduced subsequently in [EGL83] with applications to contract signing protocols: One-out-of-two Bit Oblivious Transfer, denoted $\left(\begin{array}{l}2 \\ 1\end{array}\right)$ OT, concerns the case $k=1$ in which $w_{0}$ and $w_{1}$ are single-bit secrets, generally called $b_{0}$ and $b_{1}$ in that case.

Techniques were introduced in [BCR86] and refined in [CS91b, BCS96] to reduce $\left(\begin{array}{l}2 \\ 1\end{array}\right)-\mathrm{OT}^{k}$ to $\left(\begin{array}{l}2 \\ 1\end{array}\right)-\mathrm{OT}$ : several two-party protocols were given to achieve One-out-of-two String Oblivious Transfer based on the assumption of the availability of a protocol for the simpler One-out-of-two Bit Oblivious Transfer. The fact that $\left(\begin{array}{l}2 \\ 1\end{array}\right)-O \mathrm{~T}^{k}$ can be reduced to $\left(\begin{array}{l}2 \\ 1\end{array}\right)-\mathrm{OT}$ is not surprising because a number of authors [Kil88, Cré89, CGT95] have shown that $\left(\begin{array}{l}2 \\ 1\end{array}\right)$-OT is sufficient to implement any two-party computation. Our interest in direct reductions is their far greater efficiency. With the exception of [CS91a], all previous direct reductions that we are aware of [BCR86, CS91b, BCS96] are based on a notion called zigzag functions, whose construction is reduced to finding particular types of error-correcting codes called self-intersecting codes. In a nutshell, this approach consists in selecting once and for all a suitable function $f$ from $\{0,1\}^{n}$ to $\{0,1\}^{k}$ for $n$ as small as possible $(n>k)$, so that if $x_{0}$ is a random preimage of $w_{0}$ and $x_{1}$ is a random preimage of $w_{1}$, and if $\mathcal{B}$ is given to choose via $\left(\begin{array}{l}2 \\ 1\end{array}\right)$-OT to see the $i^{\text {th }}$ bit of either $x_{0}$ or $x_{1}, 1 \leq i \leq n$, then no information can be inferred on at least one of $w_{0}$ or $w_{1}$. This approach has led to various reductions with expansion factors $\beta$ ranging from 4.8188 to 18 , that is various polynomial-time constructible methods using $n=\beta k$ instances of $\left(\begin{array}{l}2 \\ 1\end{array}\right)$-OT to perform one $\left(\begin{array}{l}2 \\ 1\end{array}\right)-\mathrm{OT}^{k}$ on $k$-bit strings. Komlós proved that this approach cannot yield an expansion factor $\beta$ that is asymptotically better than 3.5277 [CL85]. It was recently proven by Stinson that the same bound applies even to non-linear zigzags [Sti97].

The current paper exploits a new approach to this problem using privacy amplification, a notion first introduced in the context of key exchange protocols [BBR88]. The new approach allows for a solution requiring only slightly more than $2 k$ instances of $\left(\begin{array}{l}2 \\ 1\end{array}\right)$-OT to perform one $\left(\begin{array}{l}2 \\ 1\end{array}\right)-\mathrm{OT}^{k}$, and it can be extended to a whole range of generalizations of $\left(\begin{array}{l}2 \\ 1\end{array}\right)$-OT that could not be used with the reductions based on zigzag functions.

An application of the simplest of our generalizations is also considered: $\left(\begin{array}{l}2 \\ 1\end{array}\right)-\mathrm{OT}^{k}$ from $\mathcal{A}$ to $\mathcal{B}$ can be reduced to $\left(\begin{array}{l}2 \\ 1\end{array}\right)-\mathrm{OT}$ in the other direction (from $\mathcal{B}$ to $\mathcal{A}$ ) by only doubling the cost of reducing to $\left(\begin{array}{l}2 \\ 1\end{array}\right)-\mathrm{OT}$ from $\mathcal{A}$ to $\mathcal{B}$. This improves on an earlier result of [CS91a]. 


\section{Privacy Amplification Method}

Assume $\mathcal{A}$ knows a random $n$-bit string $x$ about which $\mathcal{B}$ has partial information. Privacy amplification is a technique invented in [BBR88] and refined in [BBCM95] that allows $\mathcal{A}$ to shrink $x$ to a shorter string $w$ about which $\mathcal{B}$ has an arbitrarily small amount of information even if he knows the recipe used by $\mathcal{A}$ to transform $x$ into $w$. Intuitively, this can be used to implement $\left(\begin{array}{l}2 \\ 1\end{array}\right)-\mathrm{OT}^{k}\left(w_{0}, w_{1}\right)(c)$ from $\left(\begin{array}{l}2 \\ 1\end{array}\right)$-OT because $\mathcal{A}$ can offer $\mathcal{B}$ to read one of two random strings $x_{0}$ or $x_{1}$ by a simple sequence of $\left(\begin{array}{l}2 \\ 1\end{array}\right)$-OT $\left(x_{0}^{i}, x_{1}^{i}\right)\left(c_{i}\right)$. Subsequently, $\mathcal{A}$ tells $\mathcal{B}$ how to transform $x_{0}$ into $w_{0}$ and $x_{1}$ into $w_{1}$ by way of privacy amplification. An honest $\mathcal{B}$ who accessed all the bits of $x_{c}$ can reconstruct $w_{c}$ from this information. But a dishonest $\tilde{\mathcal{B}}$ who tried to access some of the bits of $x_{0}$ and some of the bits of $x_{1}$ will not have enough information on at least one of them to infer any information on the corresponding $w_{i}$ or even joint information on both $w_{0}$ and $w_{1}$.

An important fact about the method based on zigzag functions considered in earlier papers is that there is no way for $\mathcal{B}$ to learn information about both $w_{0}$ and $w_{1}$ even though the zigzag function is known before he gets to choose which bits of $x_{0}$ and $x_{1}$ to obtain through the $\left(\begin{array}{l}2 \\ 1\end{array}\right)$-OT instances. In the new approach based on privacy amplification, $\mathcal{A}$ reveals the function to $\mathcal{B}$ after the necessary $\left(\begin{array}{l}2 \\ 1\end{array}\right)$-OT's have been performed. This allows for a protocol that is simpler, more general and more efficient, but at the cost of a vanishingly small probability of failure. A drawback of this approach is that a new function must be generated and transmitted at each run of the protocol.

The following table compares the efficiency of the earlier methods to that of privacy amplification. The column "expansion factor" gives a number $\beta$ so that a $\left(\begin{array}{l}2 \\ 1\end{array}\right)-\mathrm{OT}^{k}$ can be achieved with $\beta k$ instances of $\left(\begin{array}{l}2 \\ 1\end{array}\right)-\mathrm{OT}, s$ is a safety parameter, and $\varepsilon$ is arbitrarily small in the limit of large $k$, Thus we see that the privacy amplification method is preferable provided a probability of failure can be tolerated.

\begin{tabular}{|c|c|c|c|}
\hline Method & $\begin{array}{c}\text { expansion } \\
\text { factor }\end{array}$ & $\begin{array}{c}\text { failure } \\
\text { probability }\end{array}$ & $\begin{array}{c}\text { construction } \\
\text { time }\end{array}$ \\
\hline Monte Carlo Zigzag ${ }^{1}$ & $4.8188+\varepsilon$ & $\overline{2^{-5}}$ & $\overline{O\left(k^{2}\right)}$ \\
\hline Las Vegas Zigzag $^{2}$ & $9.6377+\varepsilon$ & 0 & $O\left(k^{2}\right)$ \\
\hline Zigzag à la Justesen ${ }^{3}$ & 18 & 0 & $O\left(k^{4}\right)$ \\
\hline Zigzag à la Goppa & 6.4103 & 0 & $O\left(k^{32}\right)$ \\
\hline Privacy Amplification & $2+\varepsilon$ & $2^{-3}$ & $\overline{O\left(k^{2}\right)}$ \\
\hline
\end{tabular}

\footnotetext{
${ }^{1}$ Attributed to Cohen and Lempel in [BCS96].

2 Attributed to Joe Kilian in [BCS96].

${ }^{3}$ From [BCS96].

${ }^{4}$ From [CZ94] based on a method of [CS91b].
} 


\section{The New Protocol}

Let $s$ be a security parameter chosen by $\mathcal{A}$ and $\mathcal{B}$ so that they agree to tolerate a probability $2^{-s}$ of failure. Let $\gamma$ be a constant to be determined later, let $n=\gamma k+s$, and let $\mathcal{F}_{2}$ denote the field of integers modulo 2 .

Privacy amplification is based on the general notion of universal classes of hash functions [CW79]. For sake of simplicity, we use a specific class of hash functions in our protocol to implement $\left(\begin{array}{l}2 \\ 1\end{array}\right)-\mathrm{OT}^{k}$ from $\left(\begin{array}{l}2 \\ 1\end{array}\right)-\mathrm{OT}$ :

$$
\left\{h \mid h(x)=M x, \text { for } M \text { a } k \times n \text { matrix over } \mathcal{F}_{2}\right\} .
$$

Other, more efficient classes of hash functions can be used, but it is not known if the definition of universal classes is sufficient in general to make our protocol work.

Protocol $3.1\left(\left(\begin{array}{l}2 \\ 1\end{array}\right)-\mathbf{O T}^{k}\left(w_{0}, w_{1}\right)(c)\right)$

1: $\mathcal{A}$ picks two random $n$-bit strings $x_{0}$ and $x_{1}$.

2: DO $_{i=1}^{n} \mathcal{A}$ transfers $t^{i} \leftarrow\left(\begin{array}{l}2 \\ 1\end{array}\right)-\mathrm{OT}\left(x_{0}^{i}, x_{1}^{i}\right)(c)$ to $\mathcal{B}$.

3: $\mathcal{A}$ picks two random $k \times n$ matrices $M_{0}$ and $M_{1}$ over $\mathcal{F}_{2}$; she announces them to $B$.

4: $\mathcal{A}$ sets $m_{0} \leftarrow M_{0} x_{0}, m_{1} \leftarrow M_{1} x_{1}, y_{0} \leftarrow m_{0} \oplus w_{0}$ and $y_{1} \leftarrow m_{1} \oplus w_{1}$; she announces $y_{0}$ and $y_{1}$ to $\mathcal{B}$.

5: $\mathcal{B}$ recovers $w_{c}$ by computing $\left(M_{c} t\right) \oplus y_{c}$.

We postpone to Sect. 5 the proof that this protocol is private provided $\gamma \geq 2$ because we shall first generalize it to permit at no extra cost the use of another primitive called XOR Oblivious Transfer. (Informally, a protocol is private if $\mathcal{B}$ cannot learn information on both $w_{0}$ and $w_{1}$ except perhaps with negligible probability. In addition, $\mathcal{B}$ must not be able to obtain joint information on $w_{0}$ and $w_{1}$ except for what follows from his a priori knowledge and his learning one of the two strings. Conversely, $\mathcal{A}$ should learn nothing at all. See [BCS96] for a formal information-theoretic definition. We shall later relax the condition to allow $\mathcal{B}$ an exponentially small amount of unauthorized information.)

\section{XOR Oblivious Transfer}

A $\left(\begin{array}{l}2 \\ 1\end{array}\right)$-XOT is an extension of $\left(\begin{array}{l}2 \\ 1\end{array}\right)$-OT that enables a sender $\mathcal{A}$ to transfer to a receiver $\mathcal{B}$ either one bit among $b_{0}$ and $b_{1}$ or their exclusive-or, at $\mathcal{B}$ 's choice. More formally, $\mathcal{A}$ inputs $b_{0}$ and $b_{1}$ into the protocol, $\mathcal{B}$ inputs $c \in\{0,1, \oplus\}$, and $\mathcal{B}$ learns $b_{c}$ while $\mathcal{A}$ learns nothing, where for convenience we use $b_{\oplus}$ to denote $b_{0} \oplus b_{1}$. As usual, this is done in an all-or-nothing fashion: $\mathcal{B}$ cannot get more information about $b_{0}$ and $b_{1}$ than $b_{0}, b_{1}$ or $b_{\oplus}$, however malicious or computationally powerful he is. Note that in our application of $\left(\begin{array}{l}2 \\ 1\end{array}\right)-\mathrm{XOT}$, which is to use it instead of 
$\left(\begin{array}{l}2 \\ 1\end{array}\right)$-OT inside Protocol 3.1, an honest $\mathcal{B}$ would never requests $b_{\oplus}$. Therefore we can safely use any protocol in which it is merely tolerated that $\mathcal{B}$ might learn $b_{\oplus}$ in cheating attempts even though $\mathcal{A}$ is not required to provide it upon request.

The $\left(\begin{array}{l}2 \\ 1\end{array}\right)$-XOT comes naturally in a specific implementation of $\left(\begin{array}{l}2 \\ 1\end{array}\right)-\mathrm{OT}$ : in [BCR86a] a protocol for $\left(\begin{array}{l}2 \\ 1\end{array}\right)-\mathrm{OT}$ is given under the assumption that deciding quadratic residuosity modulo a composite number is hard. In that implementation, the possibility that $\tilde{\mathcal{B}}$ obtains $b_{\oplus}$ arises naturally and some effort is made to prevent it. The current paper shows that this effort was unnecessary if the final goal is to implement $\left(\begin{array}{l}2 \\ 1\end{array}\right)-\mathrm{OT}^{k}$ rather than simply $\left(\begin{array}{l}2 \\ 1\end{array}\right)$-OT.

\section{Privacy}

Consider a variation of Protocol 3.1 in which the transfers at step 2 are performed through $\left(\begin{array}{l}2 \\ 1\end{array}\right)$-XOT instead of $\left(\begin{array}{l}2 \\ 1\end{array}\right)-O T$. Even though this makes no difference if $\mathcal{B}$ follows the protocol honestly, it gives him additional opportunities for cheating if he so desires. Our goal is to show that whatever program $\tilde{\mathcal{B}}$ is ran by $\mathcal{B}$, he is not able to obtain information on both $w_{0}$ and $w_{1}$, except with a probability that is exponentially small in the security parameter $s$. Moreover, it is obvious from inspection of the protocol that a cheating $\mathcal{A}$ cannot obtain any information about $\mathcal{B}$ 's secret parameter $c$. From now on, think of $x_{0}$ and $x_{1}$ as column-vectors of length $n$, and of $m_{0}=M_{0} x_{0}$ and $m_{1}=M_{1} x_{1}$ as columnvectors of length $k$, all over $\mathcal{F}_{2}$. First, we show that immediately after Step 3 of the protocol, whatever program $\tilde{\mathcal{B}}$ is ran by $\mathcal{B}$, he will have no information about one of $m_{0}$ or $m_{1}$ and no information allowing him to connect $m_{0}$ and $m_{1}$ (such as $m_{0} \oplus m_{1}$ for instance), except with exponentially small probability. (Formally, there will be some bit $\tilde{c}$ such that the first three steps of the protocol would give no additional information to $\tilde{\mathcal{B}}$ about the pair $\left(m_{0}, m_{1}\right)$ than if he were simply told the value of $m_{\tilde{c}}$; see [BCS96].) We conclude the result about $w_{0}$ and $w_{1}$ at the end of the protocol from the fact that $m_{0}$ and $m_{1}$ are used as one-time pads to transfer them.

Suppose $\tilde{\mathcal{B}}$ reads the bits $x_{c_{i}}^{i}$ with $c_{i} \in\{0,1, \oplus\}$ at his choosing. Let $g$ be a non-trivial linear function of $m_{0}$ and $m_{1}$. In other words, $g\left(m_{0}, m_{1}\right)=v_{0} m_{0} \oplus v_{1} m_{1}$ for some line-vectors $v_{0}$ and $v_{1}$ of length $k$ over $\mathcal{F}_{2}$ such that both $v_{0}$ and $v_{1}$ are non-zero ${ }^{5}$.

Theorem 1. Consider the knowledge that $\tilde{\mathcal{B}}$ has about $m_{0}$ and $m_{1}$ immediately after Step 3 of the protocol. Provided $\gamma \geq 2$,

$$
\text { Prob }\left(\exists \text { non-trivial } g \text { such that } \tilde{\mathcal{B}} \text { knows } g\left(m_{0}, m_{1}\right)\right)<2^{-s} \text {. }
$$

\footnotetext{
5 Note that by virtue of $v_{0}$ and $m_{0}$ being a line-vector and a column-vector, respectively, a "matrix" multiplication such as $v_{0} m_{0}$ computes the scalar product; similarly, given that $x_{0}$ is also a column-vector, an expression such as $v_{0} M_{0} x_{0}$ makes sense: it is simply an element of $\mathcal{F}_{2}$. This notation is handy because $v_{0} M_{0} x_{0}$ can be thought of indifferently as either the scalar product of $v_{0}$ with $M_{0} x_{0}$ or of $v_{0} M_{0}$ with $x_{0}$.
} 
Proof. We first describe the condition under which $\tilde{\mathcal{B}}$ learns $g\left(m_{0}, m_{1}\right)$ at Step 3 of the protocol for some specific non-trivial linear function $g$. By definition

$$
g\left(m_{0}, m_{1}\right)=v_{0} m_{0} \oplus v_{1} m_{1}=v_{0} M_{0} x_{0} \oplus v_{1} M_{1} x_{1}=z_{0} x_{0} \oplus z_{1} x_{1}
$$

where $z_{0}=v_{0} M_{0}$ and $z_{1}=v_{1} M_{1}$. Because $x_{0}$ and $x_{1}$ are random, $\tilde{\mathcal{B}}$ cannot learn anything about $g\left(m_{0}, m_{1}\right)$ at Step 3 unless he is lucky enough that his choices $c_{i}$ simultaneously follow

$$
c_{i}=\left\{\begin{array}{l}
0 \text { when }\left(z_{0}^{i}, z_{1}^{i}\right)=(1,0) \\
1 \text { when }\left(z_{0}^{i}, z_{1}^{i}\right)=(0,1) \\
\oplus \text { when }\left(z_{0}^{i}, z_{1}^{i}\right)=(1,1)
\end{array}\right.
$$

in all the instances of $\left(\begin{array}{l}2 \\ 1\end{array}\right)-\mathrm{XOT}$ such that $z_{0}^{i}$ and $z_{1}^{i}$ are not both 0 . (The value of $c_{i}$ is unimportant when $\left(z_{0}^{i}, z_{1}^{i}\right)=(0,0)$ since neither $x_{0}^{i}$ nor $x_{1}^{i}$ is required in that case to compute $g\left(m_{0}, m_{1}\right)$.)

But remember that $M_{0}$ and $M_{1}$ are picked at random and neither $v_{0}$ nor $v_{1}$ is zero. Therefore $z_{0}=v_{0} M_{0}$ and $z_{1}=v_{1} M_{1}$ are random binary strings of length $n$ chosen independently according to the uniform distribution. In particular, $z_{0}$ and $z_{1}$ are independent of $\tilde{\mathcal{B}}$ 's choices of $c_{i}$ 's. It follows that, for each $i$, the probability that either $\left(z_{0}^{i}, z_{1}^{i}\right)=(0,0)$ or $\tilde{\mathcal{B}}$ chose $c_{i}$ appropriately according to the above case analysis is exactly $1 / 2$. Since $\tilde{\mathcal{B}}$ must be lucky for each $i, 1 \leq i \leq n$,

$$
\text { Prob }\left(\tilde{\mathcal{B}} \text { learns } g\left(m_{0}, m_{1}\right)\right)=2^{-n}
$$

for each non-trivial linear function $g$, whatever choices $\tilde{\mathcal{B}}$ makes for the $c_{i}$ 's. Finally, given that there are less than $2^{2 k}$ such linear functions, we conclude that

$$
\begin{aligned}
& \operatorname{Prob}\left(\exists \text { non-trivial } g \text { such that } \tilde{\mathcal{B}} \text { learns } g\left(m_{0}, m_{1}\right)\right) \\
& <2^{2 k-n}=2^{(2-\gamma) k-s} \leq 2^{-s}
\end{aligned}
$$

provided $\gamma \geq 2$

Theorem 2. Protocol 3.1 is private even if the transfers at step 2 are performed through $\left(\begin{array}{l}2 \\ 1\end{array}\right)-X O T$ instead of $\left(\begin{array}{l}2 \\ 1\end{array}\right)-O T$.

Proof. We know from Theorem 1 that, except with probability at most $2^{-s}$, $\mathcal{B}$ has not learned $g\left(m_{0}, m_{1}\right)$ by the end of Step 3 for any linear function $g$ that involves both $m_{0}$ and $m_{1}$ in a non-trivial way. ${ }^{6}$ It follows that there is a $d \in\{0,1\}$ such that $\mathcal{B}$ learns no non-trivial linear function of $m_{d}$ because if he could learn non-trivial linear functions $g_{0}\left(m_{0}\right)$ and $g_{1}\left(m_{1}\right)$, he would have learned $g_{0}\left(m_{0}\right) \oplus g_{1}\left(m_{1}\right)$, a non-trivial linear function of both $m_{0}$ and $m_{1}$. We can say something stronger: not only does $\mathcal{B}$ learn no non-trivial linear function of $m_{d}$, but he learns no information of any kind that involves $m_{d}$. This is true because

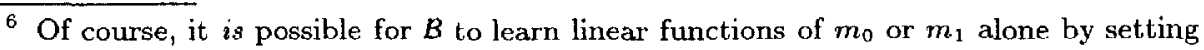
all the $c_{i}=0$ or $c_{i}=1$ as in the honest protocol. 
$m_{0}$ and $m_{1}$ are purely random and the only source of information that $\mathcal{B}$ has about them (up until Step 3) is given by linear functions of $m_{0}$ and $m_{1}$. Since $m_{d}$ is used by $\mathcal{A}$ at Step 4 as one-time pad to transmit $w_{d}$ to $\mathcal{B}$, it follows that $\mathcal{B}$ learns no information of any kind that involves $w_{d}$.

\section{Application: Reversing Oblivious Transfer}

Consider that $\mathcal{A}$ wants to send one of two words $w_{0}$ or $w_{1}$ to $\mathcal{B}$ when they only have an $\left(\begin{array}{l}2 \\ 1\end{array}\right)$-OT channel running from $\mathcal{B}$ to $\mathcal{A}$. A very efficient protocol for sending one of two bits from $\mathcal{A}$ to $\mathcal{B}$ is given in [CS91a] provided $\mathcal{A}$ does not mind the possibility that $\mathcal{B}$ might learn the exclusive-or of her two bits: two instances of reversed $\left(\begin{array}{l}2 \\ 1\end{array}\right)-$ OT are sufficient to implement $\left(\begin{array}{l}2 \\ 1\end{array}\right)$-XOT. No such efficient constructions are known that would implement $\left(\begin{array}{l}2 \\ 1\end{array}\right)$ OT from so few instances of reversed $\left(\begin{array}{l}2 \\ 1\end{array}\right)-O T$. In other words it is much easier to implement $\left(\begin{array}{l}2 \\ 1\end{array}\right)-$ XOT than $\left(\begin{array}{l}2 \\ 1\end{array}\right)-O T$ from $\mathcal{A}$ to $\mathcal{B}$ given an $\left(\begin{array}{l}2 \\ 1\end{array}\right)-O T$ channel from $\mathcal{B}$ to $\mathcal{A}$. This is fine because we just showed that $\left(\begin{array}{l}2 \\ 1\end{array}\right)$-XOT is just as good as $\left(\begin{array}{l}2 \\ 1\end{array}\right)$-OT for the purpose of implementing $\left(\begin{array}{l}2 \\ 1\end{array}\right)-\mathrm{OT}^{k}$. Therefore, $\left(\begin{array}{l}2 \\ 1\end{array}\right)-\mathrm{OT}^{k}$ from $\mathcal{A}$ to $\mathcal{B}$ can be implemented from slightly more than $4 k$ instances of $\left(\begin{array}{l}2 \\ 1\end{array}\right)-O T$ from $\mathcal{B}$ to $\mathcal{A}$. This is a three-fold improvement over [CS91a].

\section{Generalized Oblivious Transfer}

A $\left(\begin{array}{l}2 \\ 1\end{array}\right)$-GOT is a cryptographic protocol for two participants that enables a sender $\mathcal{A}$ to transfer a one-bit function evaluated on $\left(b_{0}, b_{1}\right)$ to a receiver $\mathcal{B}$ who chooses secretly which one-bit function $(f)$ he gets from her input bits. This is done in an all-or-nothing fashion: $\mathcal{B}$ cannot get more information about $b_{0}$ and $b_{1}$ than $f\left(b_{0}, b_{1}\right)$ for some $f$, however malicious or computationally powerful he is, and $\mathcal{A}$ finds out nothing about the choice $f$ of $\mathcal{B}$. As was the case with $\left(\begin{array}{l}2 \\ 1\end{array}\right)-$ XOT in Sect. 4, one may think of a $\left(\begin{array}{l}2 \\ 1\end{array}\right)$-GOT protocol as merely tolerating the fact that a cheating $\mathcal{B}$ might learn $f\left(b_{0}, b_{1}\right)$ for some $f$ rather than specifying that any such $f$ can be learned at $\mathcal{B}$ 's whim.

The following table enumerates all 14 possible non-constant functions from two bits to one. (We ignore the two constant function since they would yield no information if used.) The symbols used refer to the common boolean functions. Example: $\bar{\Lambda}$ stands for $\overline{b_{0} \wedge b_{1}}$. The notations 0 and 1 are used for the projection functions $b_{0} 0 b_{1}=b_{0}$ and $b_{0} 1 b_{1}=b_{1}$. We say that a function $f\left(b_{0}, b_{1}\right)$ is biased if the probability that $f\left(b_{0}, b_{1}\right)=1$ is not $1 / 2$ when $b_{0}$ and $b_{1}$ are chosen randomly and independently according to the uniform distribution. The ordinary $\left(\begin{array}{l}2 \\ 1\end{array}\right)-\mathrm{OT}$ is a special case of $\left(\begin{array}{l}2 \\ 1\end{array}\right)$-GOT where $\mathcal{B}$ is limited to the functions 0 and 1 . 


\begin{tabular}{|c|c|c|c|c|c|c|c|c|c|c|c|c|c|}
\hline$b_{0}$ & $b_{1}$ & $V$ & $F$ & $\Rightarrow$ & & & & & & $\rightarrow$ & & & $\mathrm{V}$ \\
\hline 0 & 0 & 1 & 0 & 0 & & $\overline{0}$ & 1 & 0 & {$[\overline{0}$} & 1 & 0 & 1 & 0 \\
\hline 1 & 0 & 0 & 1 & 0 & 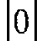 & 1 & 1 & 0 & \begin{tabular}{l|l}
0 & 1
\end{tabular} & 1 & 0 & 0 & 1 \\
\hline 0 & 0 & 0 & & 1 & 1 & 1 & 1 & 0 & 00 & 0 & 1 & 1 & 1 \\
\hline 1 & 0 & 0 & 0 & 0 & 0 & & 0 & 1 & 11 & 1 & 1 & 1 & 1 \\
\hline $\mathrm{bi}$ & & & $\bar{V}$ & $\sqrt{ }$ & & & & & & 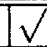 & & & 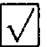 \\
\hline
\end{tabular}

It has been shown in [BCR86] that $\left(\begin{array}{l}2 \\ 1\end{array}\right)$-GOT is a sufficient primitive to implement $\left(\begin{array}{l}2 \\ 1\end{array}\right)-\mathrm{OT}$. The reduction they presented uses $\Theta(s)$ runs of $\left(\begin{array}{l}2 \\ 1\end{array}\right)-\mathrm{GOT}$ to achieve a single $\left(\begin{array}{l}2 \\ 1\end{array}\right)-\mathrm{O}$ T in such a way that the reduction may fail and give both bits to $\mathcal{B}$ with probability $2^{-s}$. If this protocol is combined with a standard reduction of $\left(\begin{array}{l}2 \\ 1\end{array}\right)-\mathrm{OT}^{k}$ we obtain a global cost of $\Theta(k s)$ runs of $\left(\begin{array}{l}2 \\ 1\end{array}\right)$-GOT per $\left(\begin{array}{l}2 \\ 1\end{array}\right)-\mathrm{OT}^{k}$. Contrary to reductions to $\left(\begin{array}{l}2 \\ 1\end{array}\right)-\mathrm{OT}$, reductions to $\left(\begin{array}{l}2 \\ 1\end{array}\right)-\mathrm{GOT}$ must involve a failure probability since it is always possible to get all the information sent by $\mathcal{A}$ by selecting the appropriate biased function at each transfer by sheer luck. For example, if $\mathcal{B}$ requests $x_{0}^{i} \wedge x_{1}^{i}$ at step 2 of Protocol 3.1 for some $i$, and if he obtains the value 1 , then he knows that both $x_{0}^{i}$ and $x_{1}^{i}$ are equal to 1 . Using the new privacy amplification method we obtain a direct reduction of $\left(\begin{array}{l}2 \\ 1\end{array}\right)-\mathrm{OT}^{k}$ at a cost of only $\Theta(k+s)$ instances of $\left(\begin{array}{l}2 \\ 1\end{array}\right)$-GOT.

Consider a variation of Protocol 3.1 in which the transfers of step 2 are performed through $\left(\begin{array}{l}2 \\ 1\end{array}\right)$-GOT instead of $\left(\begin{array}{l}2 \\ 1\end{array}\right)$-OT. Our goal is to show that whatever program $\tilde{\mathcal{B}}$ is ran by $\mathcal{B}$, he is not able to obtain non-negligible information on both $w_{0}$ and $w_{1}$, except with a probability that is exponentially small in the security parameter $s$. Contrary to the analysis in Sect. 5 , it will no longer suffice to take $n=\gamma k+s$ for some $\gamma$, but $n$ will nevertheless remain in $\Theta(k+s)$-see the proof of Theorem 3 for details. First we show that immediately after Step 3 of the protocol, whatever program $\tilde{\mathcal{B}}$ is $\operatorname{ran}$ by $\mathcal{B}$, he will have negligible information about one of $m_{0}$ or $m_{1}$, and negligible information allowing him to connect $m_{0}$ and $m_{1}$. We conclude a similar result about $w_{0}$ and $w_{1}$ at the end of the protocol from the fact that $m_{0}$ and $m_{1}$ are used as one-time pads to transfer them.

Suppose $\tilde{\mathcal{B}}$ obtains bits $x_{c_{i}}^{i}$ with $c_{i} \in\{\bar{\nabla}, \mp, \overline{1}, \rightrightarrows, \overline{0}, \oplus, \bar{\wedge}, \wedge, \bar{\oplus}, 0, \rightarrow, 1, \leftarrow, \vee\}$ at his choosing. As before, let $g$ be a non-trivial linear function of $m_{0}$ and $m_{1}$, that is $g\left(m_{0}, m_{1}\right)=v_{0} m_{0} \oplus v_{1} m_{1}$ for some non-zero binary line-vectors $v_{0}$ and $v_{1}$ of length $k$. We say that $\mathcal{B}$ can $\alpha$-bias a bit if he can guess it with probability better than $\frac{1}{2}+\alpha$ of being correct.

Theorem 3. Consider the knowledge that $\tilde{\mathcal{B}}$ has about $m_{0}$ and $m_{1}$ immediately after Step 3 of the protocol.

$$
\text { Prob }\left(\exists \text { non-trivial } g \text { such that } \tilde{\mathcal{B}} \text { can } 2^{-k-1-s / 2} \text {-bias } g\left(m_{0}, m_{1}\right)\right)<2^{-s}
$$

provided $n$ is chosen appropriately in $\Theta(k+s)$. 
Proof. Let $\gamma$ and $a$ be constants to be determined later and let $n=(a+1)(\gamma k+s)$. Let Biased $=\left\{i \mid c_{i} \in\{\bar{\nabla}, F, \rightrightarrows, \bar{\wedge}, \wedge, \rightarrow, \leftarrow, \vee\}\right\}$, the set of positions where $\mathcal{B}$ uses a biased function. If \#Biased $<a(\gamma k+s)$ then Theorem 1 applies with $\gamma \geq 2$ and $n=\gamma k+s$. We thus get the desired result. Otherwise \#Biased $\geq a(\gamma k+s)$ is the more interesting case to consider. Consider the set of positions where $\mathcal{B}$ has used a biased function. As before, $\tilde{\mathcal{B}}$ would have learned $g\left(m_{0}, m_{1}\right)$ exactly if he had simultaneously obtained

$$
\begin{aligned}
& x_{0}^{i} \text { when }\left(z_{0}^{i}, z_{1}^{i}\right)=(1,0) \\
& x_{1}^{i} \text { when }\left(z_{0}^{i}, z_{1}^{i}\right)=(0,1) \\
& x_{\oplus}^{i} \text { when }\left(z_{0}^{i}, z_{1}^{i}\right)=(1,1)
\end{aligned}
$$

for all $i$ for which $z_{0}^{i}$ and $z_{1}^{i}$ are not both 0 .

Remember that $M_{0}, M_{1}, x_{0}$ and $x_{1}$ are picked at random. Thus $z_{0}$ and $z_{1}$ are random binary words of length $n$. Since $\mathcal{B}$ has used a biased function in position $i$, with probability $1 / 4$ he will have learned both $x_{0}^{i}$ and $x_{1}^{i}$, and with probability $3 / 4$ he will be able to $1 / 6$-bias $x_{0}^{i}, x_{1}^{i}$ and $x_{\oplus}^{i}$. (This is because each biased function has one output that uniquely defines a specific pair of inputs, while the other output leaves three pairs of inputs equally likely.) This means that in each such position $i, \tilde{\mathcal{B}}$ has obtained the bit he needs with probability $7 / 16$ and with probability $9 / 16$ he can only $1 / 6$-bias the bit he needs. Of the $a(\gamma k+s)$ such values of $i$, less than $a(\gamma k+s) / 4$ of them will fall in the second case with probability at most $2 e^{-25 a(\gamma k+s) / 1024} \approx 2^{-(\gamma k+s)}$ according to Bernstein's law of large numbers [Rén70, Chap. VII, Sect. 4, Theorem 2], for $a \approx 28$. When $7(\gamma k+s)$ of the bits involved in the calculation of $g\left(m_{0}, m_{1}\right)$ are 1/6-biased, even if all the other bits are exactly known, $\mathcal{B}$ can only $(1 / 3)^{7(\gamma k+s)} / 2$-bias the value of $g\left(m_{0}, m_{1}\right)$. (In general, $\delta$-biasing each of $x_{1}, x_{2}, \ldots, x_{l}$ allows to $(2 \delta)^{l} / 2$-bias $x_{1} \oplus x_{2} \oplus \ldots \oplus x_{l}$ [Cré90].) It follows that for any set of choices $\left\{c_{i}\right\}$, and any $v_{0}, v_{1} \neq 0^{k}$

$$
\operatorname{Prob}\left(\overline{\mathcal{B}} \text { can } 3^{-7(\gamma k+s)} / 2-\text { bias } g\left(m_{0}, m_{1}\right)\right)<2^{-(\gamma k+s)} .
$$

Finally, given that there are less than $2^{2 k}$ pairs $v_{0}, v_{1}$, taking $\gamma \geq 2$, and using the fact that $3^{-7(\gamma k+s)} / 2 \leq 2^{-k-1-s / 2}$, we conclude as desired that

$$
\begin{aligned}
& \text { Prob }\left(\exists \text { non-trivial } g \text { such that } \tilde{\mathcal{B}} \text { can } 2^{-k-1-s / 2} \text {-bias } g\left(m_{0}, m_{1}\right)\right) \\
& <2^{2 k} 2^{-(\gamma k+s)} \leq 2^{-s} .
\end{aligned}
$$

To conclude that, except with probability $2^{-s}, \mathcal{B}$ has no more than $2^{-s}$ bit of information on at least one of $m_{0}$ or $m_{1}$ immediately after Step 3 , and therefore no more than $2^{-s}$ bit of information on at least one of $w_{0}$ or $w_{1}$ at the end of the protocol (even if he is given the other string-see the Appendix for formal definitions), it suffices to apply the following theorem with $\varepsilon=1 / 2^{k+1+s / 2}$. 
Theorem 4. Let $k$ be an integer and $\varepsilon \leq 1 / 2^{k+1}$. Consider a $k$-bit string $m$ so that $\mathcal{B}$ cannot $\varepsilon$-bias any non-trivial linear function of the bits of $m$. Then $\mathcal{B}$ 's information on $m$ in the sense of Shannon is less than $\left(2^{k+1} \varepsilon\right)^{2}$ bit.

Proof sketch. Let $X$ be the random variable over the binary strings of length $k$ that corresponds to $\mathcal{B}$ 's probability distribution on $m$. Consider the set $G$ of all non-trivial linear functions on $k$-bit strings: there are exactly $2^{k}-1$ such functions. For any $g \in G$, let $p_{g}$ be the probability that $g(X)=0$. We have $\frac{1}{2}-\varepsilon<p_{g}<\frac{1}{2}+\varepsilon$ for all $g \in G$ by assumption that $\mathcal{B}$ cannot $\varepsilon$-bias non-trivial linear functions of the bits of $m$.

It is easily shown that the probability that $X=x$ for any given string $x$ is given by

$$
\operatorname{Prob}(X=x)=2^{-k}+\frac{1}{2^{k}} \sum_{g \in G} s(g, x) \times\left(2 p_{g}-1\right)
$$

for some function $s: G \times\{0,1\}^{k} \rightarrow\{-1,1\}$ whose detail does not concern us. It follows that $\operatorname{Prob}(X=x)$ differs from $2^{-k}$ by less than the largest value of $2 p_{g}-1$ in absolute value, which is less than $2 \varepsilon$. The random variable $X$ that would give the most information to $\mathcal{B}$, yet respect the above constraint, would have half the strings with probability $2^{-k}-2 \varepsilon$ and the other half with probability $2^{-k}+2 \varepsilon$. Therefore,

$$
\begin{aligned}
\mathbf{H}(X) & \leq-2^{k-1}\left(2^{-k}-2 \varepsilon\right) \lg \left(2^{-k}-2 \varepsilon\right)-2^{k-1}\left(2^{-k}+2 \varepsilon\right) \lg \left(2^{-k}+2 \varepsilon\right) \\
& =\left(\frac{\left(2^{k+1} \varepsilon\right)^{2}}{1 \times 2}+\frac{\left(2^{k+1} \varepsilon\right)^{4}}{3 \times 4}+\frac{\left(2^{k+1} \varepsilon\right)^{6}}{5 \times 6}+\frac{\left(2^{k+1} \varepsilon\right)^{8}}{7 \times 8}+\cdots\right) / \ln 2 \\
& <\left(2^{k+1} \varepsilon\right)^{2} .
\end{aligned}
$$

\section{Open Problems}

The value of $n$ used in our proof of Theorem 3 is in $\Theta(k+s)$ but we conjecture that it could be made significantly smaller in terms of the hidden constant, perhaps as small as $2 k+s$.

As a further generalization, consider any $\alpha<2$. An $\alpha-\left(\begin{array}{l}2 \\ 1\end{array}\right)-$ UOT is a cryptographic protocol for two participants that enables a sender $\mathcal{A}$ to transfer $\alpha$ bits of information, in the sense of Shannon, about two bits $\left(b_{0}, b_{1}\right)$ to a receiver $\mathcal{B}$ who chooses secretly which information $\Omega_{\left(b_{0}, b_{1}\right)}$ he gets from her input bits. We require that $\Omega_{(x, y)}$ be a random variable such that $\mathbf{H}\left(\left(B_{0}, B_{1}\right) \mid \Omega_{\left(B_{0}, B_{1}\right)}\right) \geq 2-\alpha$ when $B_{0}$ and $B_{1}$ are uniformly distributed over $\{0,1\}$. This is done in an all-or-nothing fashion: $\mathcal{B}$ cannot get more information about $b_{0}$ and $b_{1}$ than a sample from $\Omega_{\left\{b_{0}, b_{1}\right\}}$ for some $\Omega$, however malicious or computationally powerful he is, and that $\mathcal{A}$ finds out nothing about the choice $\Omega$ 
of $\mathcal{B}$. To see that this is genuinely more general than $\left(\begin{array}{l}2 \\ 1\end{array}\right)-\mathrm{GOT}$, consider the case in which $\mathcal{B}$ would request to see both bits through a binary symmetric channel with error rate $11 \%$. Because $\mathbf{H}_{2}(11 \%) \approx 0.5$, this would give $\mathcal{B}$ one bit of information about the two bits of $\mathcal{A}$. However, this scenario cannot be simulated with $\left(\begin{array}{l}2 \\ 1\end{array}\right)-\mathrm{GOT}$.

Conjecture 5. For all $\alpha<2$ (or perhaps merely for all $\alpha \leq 1$ ?), Protocol 3.1 remains private even if occurrences of $\left(\begin{array}{l}2 \\ 1\end{array}\right)-O T$ are replaced with $\alpha-\left(\begin{array}{l}2 \\ 1\end{array}\right)-U O T$, provided $n \geq \beta_{\alpha}(k+s)$ for an appropriate constant $\beta_{\alpha}$ to be determined, where $s$ is the safety parameter.

Conjecture 6. If conjecture 5 fails as stated, it works if Shannon entropy is replaced with Rényi entropy of order $\rho$ in the definition of $\alpha-\left(\begin{array}{l}2 \\ 1\end{array}\right)$-UOT for all $\rho>1$ [Cac97] or perhaps merely for $\rho=2$ [BBCM95].

\section{Acknowledgements}

We thank Dominic Mayers and Louis Salvail for their help, comments, suggestions and support.

\section{References}

[BBCM95] C.H. Bennett, G. Brassard, C. Crépeau and U.M. Maurer, "Generalized privacy amplification", IEEE Transaction on Information Theory, Vol. 41, no. 6, November 1995, pp. 1915 - 1923.

[BBR88] C.H. Bennett, G. Brassard and J.-M. Robert, "Privacy amplification by public discussion", SIAM Journal on Computing, Vol. 17, no. 2, April 1988, pp. $210-229$.

[BCR86] G. Brassard, C. Crépeau and J.-M. Robert, "Information theoretic reductions among disclosure problems", Proceedings of 27th Annual IEEE Symposium on Foundations of Computer Science, 1986, pp. 168-173.

[BCR86a] G. Brassard, C. Crépeau and J.-M. Robert, "All-or-nothing disclosure of secrets", Advances in Cryptology: Proceedings of Crypto '86, Springer-Verlag, 1987, pp. $234-238$.

[BCS96] G. Brassard, C. Crépeau and M. Sántha, "Oblivious transfers and intersecting codes", IEEE Transactions on Information Theory, Vol. 42, no. 6, November 1996, pp. 1769-1780.

[Cac97] C. Cachin, "Smooth entropy and Rényi entropy", Advances in Cryptology: Proceedings of Eurocrypt '97, Springer-Verlag, 1997.

[CW79] J.L. Carter and M.N. Wegman, "New hash functions and their use in authentication and set equality", Journal of Computer and System Sciences, Vol. 22, 1981, pp. 265-279.

[CL85] G. D. Cohen and A. Lempel, "Linear intersecting codes", Discrete Mathematics, Vol. 56, 1985, pp. 35-43. 
[CZ94] G. D. Cohen and G. Zémor, "Intersecting codes and independent families", IEEE Transactions on Information Theory, Vol. 40, no. 6, November 1994, pp. $1872-1881$.

[Cré89] C. Crépeau, "Verifiable disclosure of secrets and application", Advances in Cryptology: Proceedings of Eurocrypt '89, Springer-Verlag, 1990, pp. $181-191$.

[Cré90] C. Crépeau, Correct and Private Reductions Among Oblivious Transfers, PhD thesis, Department of Electrical Engineering and Computer Science, Massachusetts Institute of Technology, 1990. Supervised by Silvio Micali.

[CGT95] C. Crépeau, J. van de Graaf and A. Tapp, "Committed oblivious transfer and private multi-party computations", Advances in Cryptology: Proceedings of Crypto '95, Springer-Verlag, 1995, pp. 110-123.

[CS91a] C. Crépeau and M. Sántha, "On the reversibility of oblivious transfer", Advances in Cryptology: Proceedings of Eurocrypt '91, Springer-Verlag, 1991, pp. 106-113.

[CS91b] C. Crépeau and M. Sántha, "Efficient reductions among oblivious transfer protocols based on new self-intersecting codes", Sequences II, Methods in Communications, Security and Computer Science, Springer-Verlag, 1991, pp. $360-368$.

[EGL83] S. Even, O. Goldreich and A. Lempel, "A randomized protocol for signing contracts", Proceedings of Crypto 82, Plenum Press, New York, 1983, pp. $205-210$.

[GMR89] S. Goldwasser, S. Micali and C. Rackoff, "The knowledge complexity of interactive proof-systems", SIAM Journal on Computing, Vol. 18, 1989, pp. $186-208$.

[Kil88] J. Kilian, "Founding cryptography on oblivious transfer", Proceedings of 20th Annual ACM Symposium on Theory of Computing, 1988, pp. $20-31$.

[Rab81] M. O. Rabin, "How to exchange secrets by oblivious transfer", Technical Memo TR-81, Aiken Computation Laboratory, Harvard University, 1981.

[Rén70] A. Rényi, Probability Theory, North Holland, 1970.

[Sti97] D. R. Stinson, Private communication, 12 February 1997.

[Wie70] S. Wiesner, "Conjugate coding", Sigact News, Vol. 15, no. 1, 1983, pp. 78-88. Original manuscript written circa 1970. 


\section{A Appendix: Information Theoretic Definition of Generalized Oblivious Transfer}

A cryptographic protocol is a multi-party synchronous program that describes for each party the computations to be performed or the messages to be sent to some other party at each point in time. The protocol terminates when no party has any message to send or information to compute. The protocols we describe in this paper all take place between two parties $\mathcal{A}$ and $\mathcal{B}$. We denote by $\overline{\mathcal{A}}$ and $\overline{\mathcal{B}}$ the honest programs to be executed by $\mathcal{A}$ and $\mathcal{B}$ : honest parties behave according to $\mathcal{A}$ and $\overline{\mathcal{B}}$ and no other program. In the following definitions of correctness and privacy we also consider alternative dishonest programs $\tilde{\mathcal{A}}$ and $\tilde{\mathcal{B}}$ executed by $\mathcal{A}$ or $\mathcal{B}$ in a effort to obtain unauthorized information from one another. The definitions specify the result of honest parties interacting together through a specific protocol as well as the possible information leakage of an honest party facing a dishonest party. We are not concerned with the situation where both parties may be dishonest as they can do anything they like in that case; we are only concerned with protecting an honest party against a dishonest party. At the end of each execution of a protocol, each party will issue an "accept" or "reject" verdict regarding their satisfaction with the behaviour of the other party. Two honest parties should always issue "accept" verdicts at the end of their interactions. $\Lambda \mathrm{n}$ honcst party will issue a "reject" verdict at the end of a protocol if he received some message from the other party of improper format or some message not satisfying certain conditions specified by the protocol. We also implicitly assume certain time limits for each party to issue messages to each other: after a specified amount of time a party will give up interacting with the other party and issue a "reject" verdict.

As discussed in Sect. 7, a $\left(\begin{array}{l}2 \\ 1\end{array}\right)$-GOT is a cryptographic protocol for two participants that enables a sender $\mathcal{A}$ to transfer a one-bit function of two bits $b_{0}$ or $b_{1}$ to a receiver $\mathcal{B}$ who chooses secretly which function $f\left(b_{0}, b_{1}\right)$ he gets. This is done in an all-or-nothing fashion, which means that $\mathcal{B}$ cannot get partial information about $b_{0}$ and $b_{1}$ at the same time, however malicious or computationally powerful he is, and that $\mathcal{A}$ finds out nothing about the choice $f$ of $\mathcal{B}$.

Formally speaking we describe a two-party protocol that satisfies the following constraints of correctness and privacy, similar to those introduced for $\left(\begin{array}{l}2 \\ 1\end{array}\right)$-OT in [BCS96].

Let $\left[P_{0}, P_{1}\right](a)(b)$ be the random variable (since $P_{0}$ and $P_{1}$ may be probabilistic programs) that describes the outputs obtained by $\mathcal{A}$ and $\mathcal{B}$ when they execute together the programs $P_{0}$ and $P_{1}$ on respective inputs $a$ and $b$. Similarly, let $\left[P_{0}, P_{1}\right]^{*}(a)(b)$ be the random variable that describes the total information (including not only messages received and issued by the partics but also the result of any local random sampling they may have performed) acquired during the execution of protocol $\left[P_{0}, P_{1}\right]$ on inputs $a$ and $b$. Let $\left[P_{0}, P_{1}\right]_{P}(a)(b)$ and $\left[P_{0}, P_{1}\right]_{P}^{*}(a)(b)$ be the marginal random variables obtained by restricting the above to only one party $P$. The latter is often called the view of $P$ [GMR89]. 
In the following definition, the equality sign $(\Rightarrow)$ means that the distributions on the 1.h.s. and the r.h.s. are the same. When required, we shall use more flexible definitions that would allow an exponentially small probability of failure or amount of unauthorized information leakage. Details are left to the reader.

Definition 7 (Correctness). Protocol $[\overline{\mathcal{A}}, \overline{\mathcal{B}}]$ is correct for $\left(\begin{array}{l}2 \\ 1\end{array}\right)$-GOT if

$-\forall b_{0}, b_{1} \in\{0,1\}, f:\{0,1\}^{2} \rightarrow\{0,1\}$

$$
[\overline{\mathcal{A}}, \overline{\mathcal{B}}]\left(b_{0}, b_{1}\right)(f)=\left(\epsilon, f\left(b_{0}, b_{1}\right)\right)
$$

- for any program $\tilde{\mathcal{A}}$ there exists a probabilistic program $\tilde{\mathcal{A}}^{\prime}$ s.t. $\forall b_{0}, b_{1} \in\{0,1\}, f:\{0,1\}^{2} \rightarrow\{0,1\}$

$$
[\tilde{\mathcal{A}}, \overline{\mathcal{B}}]_{\overline{\mathcal{B}}}\left(b_{0}, b_{1}\right)(f) \mid \overline{\mathcal{B}} \text { accepts }=[\overline{\mathcal{A}}, \overline{\mathcal{B}}]_{\overline{\mathcal{B}}}\left(\tilde{\mathcal{A}}^{\prime}\left(b_{0}, b_{1}\right)\right)(f) \mid \overline{\mathcal{B}} \text { accepts }
$$

Intuitively, condition (1) means that if the protocol is executed as described, it will accomplish the task it was designed for: $\mathcal{B}$ receives bit $f\left(b_{0}, b_{1}\right)$ and $\mathcal{A}$ receives nothing. Condition (2) means that in situations in which $\mathcal{B}$ does not abort, $\mathcal{A}$ cannot induce a distribution on $\mathcal{B}$ 's output using a dishonest $\tilde{\mathcal{A}}$ that she could not induce simply by changing the input words and then being honest.

Let $B_{0}, B_{1}$ and $F$ be the random variables taking values over $\{0,1\}$ and $\{0,1\}^{2} \rightarrow\{0,1\}$ that describe $\mathcal{A}$ 's and $\mathcal{B}$ 's inputs. We assume that both $\mathcal{A}$ and $\mathcal{B}$ are aware of the joint probability distribution of these random variables $P_{B_{0}, B_{1}, F}$. A sample $b_{0}, b_{1}, f$ is generated from that distribution and $b_{0}, b_{1}$ is provided as $\mathcal{A}$ 's secret input while $f$ is provided as $\mathcal{B}$ 's secret input.

Definition 8 (Privacy). Protocol $[\overline{\mathcal{A}}, \overline{\mathcal{B}}]$ is private for $\left(\begin{array}{l}2 \\ 1\end{array}\right)-\mathrm{GOT}$ if $\forall B_{0}, B_{1} \in\{0,1\}, F:\{0,1\}^{2} \rightarrow\{0,1\}$

- $\forall b_{0}, b_{1} \in\{0,1\}$ and for any program $\tilde{\mathcal{A}}$

$$
\mathbf{I}\left(F ;[\tilde{\mathcal{A}}, \overline{\mathcal{B}}]_{\mathcal{A}}^{*}\left(B_{0}, B_{1}\right)(F) \mid\left(B_{0}, B_{1}\right)=\left(b_{0}, b_{1}\right)\right)=0
$$

- $\forall f:\{0,1\}^{2} \rightarrow\{0,1\}$ and for any program $\tilde{\mathcal{B}}$ there exists a random variable $\tilde{F}=\Omega(F):\{0,1\}^{2} \rightarrow\{0,1\}$ s.t.

$$
\mathbf{I}\left(\left(B_{0}, B_{1}\right) ;[\overline{\mathcal{A}}, \tilde{\mathcal{B}}]_{\mathcal{B}}^{*}\left(B_{0}, B_{1}\right)(F) \mid F=f, \tilde{F}\left(B_{0}, B_{1}\right)\right)=0 .
$$

The above two conditions are designed to guarantee that each party is limited to the information he or she should get according to the honest task definition. Condition (3) means that $\tilde{\mathcal{A}}$ cannot acquire any information about $F$ through the protocol. On the other hand, condition (4) means that $\tilde{\mathcal{B}}$ may acquire only one bit of deterministic information about $B_{0}, B_{1}$ through the protocol. We do not require that $\tilde{\mathcal{B}}$ be given $F\left(B_{0}, B_{1}\right)$ because there is no way to prevent him from obtaining any other $\tilde{F}\left(B_{0}, B_{1}\right)$ through otherwise honest use of the protocol. 